\title{
ELASTICITY ANALYSIS OF SANDWICH PIPES
}

\section{WITH FUNCTIONALLY GRADED INTERLAYERS}

\author{
Roberta Sburlati \\ Department of Civil, Chemical and Environmental Engineering, \\ University of Genova, Via Montallegro 1, 16145 Genova, Italy \\ E-mail: roberta.sburlati@unige.it
}

\begin{abstract}
Maria Kashtalyan
Centre for Micro-and Nanomechanics (CEMINACS), School of Engineering, University of Aberdeen, Fraser Noble Building, Aberdeen AB24 3UE, Scotland UK

E-mail:m.kashtalyan@abdn.ac.uk
\end{abstract}

March 12, 2016

\begin{abstract}
Sandwich pipes that combine structural performance with thermal insulation in their design are viewed as a light-weight alternative to pipe-in-pipe systems, in which the core material is used only for thermal insulation purposes. Incorporating functionally graded interlayers into the sandwich pipe design may help improve adhesion at the interfaces between the core layer and inner and outer pipes which has been identified as one of the major factors affecting sandwich pipe performance. In this paper, sandwich pipes with two thin functionally graded interlayers between the core layer and inner/outer pipes
\end{abstract}


are investigated in the context of elasticity theory. Closed form analytical solutions are derived for stresses and displacements in the pipes subjected to internal and/or external pressure. Comparative analysis of sandwich pipes with and without functionally graded interlayers is performed and beneficial effect of graded interlayers on stresses and displacements in the pipe is established.

Keywords: Functionally Graded Material; elasticity theory; sandwich pipe

\section{INTRODUCTION}

As oil and gas production move to deepwater and ultra deepwater fields new structural configurations are required to meet simultaneous demands for thermal insulation and mechanical integrity to ensure safe and reliable transportation of hydrocarbons. Since single walled pipes are not viable in these conditions due to their limited operational depths and lack of insulation, pipe-in-pipe systems have been developed over the past two decades for fields with flow assurance challenges (Bai and Bai, 2014).

A typical pipe-in-pipe system consists of an inner pipe positioned inside an outer pipe, often with the help of centralisers located at certain intervals along the inner pipe. The annular space between the inner and outer pipe is filled with insulation material to meet specific thermal requirements. The outer pipe is designed to withstand high external pressure dictated by the water depth and installation method. More recently, electrically heated pipe-in-pipe systems have been developed (Denniel, Bonneau and Savy, 2011; Denniel, 2015) which have the capability to maintain the required temperature of the fluid inside the inner pipe thus offering enhanced flow assurance.

It should be pointed out that, in the pipe-in-pipe concept, the insulation material does not perform any structural function, which is performed entirely by the outer and inner pipes. This means the annular space between the inner pipe and outer pipe is not used to its

full structural potential. With increasing water depths and associated increasing demands on structural performance, the pipe wall thickness in pipe-in-pipe systems will have to increase 
leading to pipe-in-pipe systems becoming exceedingly heavy and uneconomical.

As a lightweight alternative to pipe-in-pipe systems, a concept of sandwich pipe is being developed. A sandwich pipe combines thermal insulation and structural performance in its design and attempts to realise the full structural potential of the annular space between the inner pipe and outer pipe. A sandwich pipe typically consists of two thin-walled pipes an inner pipe and an outer pipe and a core layer that completely fills the annular space between the pipes and is bonded to them. Estefen, Netto and Pasqualino (2005) performed small-scale tests to evaluate the structural performance of sandwich pipes with two different options of core material. The obtained experimental results were used to validate a threedimensional finite element model that took into account nonlinear geometric and material behaviour. Strength analysis of sandwich pipes under combined external pressure and longitudinal bending showed that sandwich pipe systems with either cement or polypropylene cores are feasible options for ultra deepwater applications.

An analytical approach for estimating the buckling capacity of sandwich pipes with various structural configurations and core materials, subject to external hydrostatic pressure was developed by Arjomandi and Taheri (2010). In addition to the exact solution, they proposed two simplified equations for estimating the buckling capacity of two configurations commonly used in practice. Arjomandi and Taheri (2011a, b) also performed extensive finite element modelling of sandwich pipes. They analysed different bonding scenarios at the interfaces between the core layer and the pipe layers and examined the effect of material and geometrical nonlinearities on the pipe buckling and post-buckling behaviour. On the basis of a large number of finite element models, a set of simplified and practical equations for calculating the external pressure capacity of sandwich pipes was proposed. Behaviour of sandwich pipe systems under pure bending was studied by Arjomandi and Taheri (2012).

Collapse behaviour of sandwich pipes with strain hardening cementitious composite reinforced with polyvinylalcohol (PVA) fibers as a core material was investigated experimentally and numerically by An et al (2014). A parametric study examined the effects of ovality, thickness and outer/inner radius ratio on the collapse pressure of these sandwich pipes. Post- 
buckling responses and pressure capacity of sandwich pipes with the solid polypropylene core was investigated by He et al (2015) using finite element modelling. The degree of the interlayer adhesion between the core layer and the surrounding pipes was modelled by the contact surfaces adopting different maximum shear strength values to allow the relative displacement between the layers. The effects of inter-layer adhesion interactions, thickness-to-radius ratios, the core thickness, the material parameters, the relative initial ovality directions and the inelastic anisotropy on the collapse pressure of sandwich pipes were examined.

Adhesion between the core layers and the inner and outer pipes has been identified as one of the major factors affecting performance of sandwich pipes. Castello and Estefen (2007) investigated the influence of the inter-layer adhesion between steel and polymer on the ultimate strength of sandwich pipes under external pressure and longitudinal bending using finite element modelling. The effect of the reeling method of installation was also simulated. It was established that the ultimate strength of the sandwich pipe is strongly dependent on the shear stress acting at the interface between the core and the pipes. Arjomandi and Taheri (2011a) investigated elastic buckling capacity of bonded and unbonded sandwich pipes under external hydrostatic pressure and examined the influence of intra-layer adhesion configuration of the pressure capacity of sandwich pipes. Four bonding configurations were considered: core fully bonded to both pipes; core fully bonded to the inner pipe but free to slide against the outer pipe; core fully bonded to the outer pipe but free to slide against the inner pipe; core unbonded to both pipes. They established that if the core layer is free to slide against both the inner and outer pipes, the increase in the core modulus of elasticity would not improve the structural performance of the pipe when subject to external pressure. For other configurations, however, the increase in the cores modulus of elasticity would increase the buckling pressure of the system. One potential solution to the adhesion problem in sandwich pipes is to incorporate the concept of Functionally Graded Material (FGM) into the sandwich pipe design and introduce functionally graded interlayers between the core and the inner and outer pipes. Functionally Graded Materials (FGM) are heterogeneous composite materials with gradient compositional variation of the constituents from one surface of the 
material to the other which results in continuously varying material properties (Suresh and Mortensen, 1999). Functionally Graded Materials has generated a lot of interest in recent years, see for example reviews by Birman and Byrd (2007), and Jha, Kant and Singh (2013).

Beneficial effect of functionally graded interlayers on stress and displacement fields has been already established for coating/substrate systems (Kashtalyan and Menshykova, 2009; Sburlati et al, 2015), while using thin functionally graded layer was shown to reduce stresses in hollow pressurized cylinders (Sburlati, 2012) and spherical vessels (Atashipour et al, 2014) as well as around open holes (Sburlati, 2013).

The benefit of FGM elements in sandwich cylindrical shells has been recently studied also intestigate vibration and buckling using graded coating (Sofiyev, 2014) or core (Sofiyev and Kuruoglu, 2015a) or analyse dynamic instability in sandwich shells with graded interlayers (Sofiyev and Kuruoglu, 2015b).

In this paper, we examine sandwich pipes with functionally graded interlayers between the core layer and the inner/outer pipes and analyse the effect of FGM interlayers on response of sandwich pipes to internal and/or external pressure and their combination. If proven beneficial, FGM interlayers could be potentially developed for specific combinations of pipe and core materials and applied as coatings to the internal surface of the outer pipe and external surface of the inner pipe prior to the annular space being filled with the core material.

\section{ANALYTICAL MODELLING}

\subsection{Problem formulation}

Let us consider a sandwich pipe of internal radius $a$ and external radius $b$, referred to the cylindrical co-ordinate system, with $z$-axis directed along the pipe axis. The pipe, crosssection of which is shown in Fig. 1, consists of five layers: the inner pipe (layer 1) of thickness $h_{l}$, the outer pipe (layer 5) of thickness $h_{l}$, the core layer (layer 3 ) of thickness $2 h_{c}$ and two interlayers of the same thickness $t$, one being the inner interlayer (layer 2) between the inner pipe and the core layer, and another being the outer interlayer (layer 4) between the core 
layer and the outer pipe. The total thickness of the pipe wall is denoted $H=2\left(h_{l}+h_{c}+t\right)$.

The material of the inner and outer pipes is assumed to be homogeneous isotropic material with Young's modulus $E_{l}$, while the core layer is assumed to be a softer homogeneous isotropic material with Young's modulus $E_{c}$. We assume the two interlayers to be made of functionally graded material, with Young's modulus that varies with the radial co-ordinate according to the power law. In the inner interlayer (layer 2) this variation has the form

$$
E(r)=E_{l}\left(\frac{r}{a+h_{l}}\right)^{n}
$$

In the outer interlayer (layer 4)

$$
E(r)=E_{l}\left(\frac{2 a+H-r}{a+h_{l}}\right)^{n}
$$

where $n$ is the inhomogeneity parameter

$$
n=\frac{\ln \left(\frac{E_{c}}{E_{l}}\right)}{\ln \left(\frac{a+h_{l}+t}{a+h_{l}}\right)} .
$$

In this way, the same value of the inhomogeneity parameter can be used to describe the increase of Young's modulus in the outer graded interlayer and its decrease in the inner graded interlayer.

Figure 2 shows variation of Young's modulus across the wall thickness of this sandwich pipe for $E_{l}=200 E_{c}$; Poisson's ratio for all five layers of the pipe is assumed to be constant and the same.

We assume that all layers of the pipe are perfectly bonded to each other, with displace- 
ment and stress continuity conditions fulfilled at all interfaces in the form

$$
\begin{aligned}
& u^{(1)}\left(a+h_{l}\right)=u^{(2)}\left(a+h_{l}\right) \\
& \sigma_{r}^{(1)}\left(a+h_{l}\right)=\sigma_{r}^{(2)}\left(a+h_{l}\right) \\
& u^{(2)}\left(a+h_{l}+t\right)=u^{(3)}\left(a+h_{l}+t\right) \\
& \sigma_{r}^{(2)}\left(a+h_{l}+t\right)=\sigma_{r}^{(3)}\left(a+h_{l}+t\right) \\
& u^{(3)}\left(a+h_{l}+t+2 h_{c}\right)=u^{(4)}\left(a+h_{l}+t+2 h_{c}\right) \\
& \sigma_{r}^{(3)}\left(a+h_{l}+t+2 h_{c}\right)=\sigma_{r}^{(4)}\left(a+h_{l}+t+2 h_{c}\right) \\
& u^{(4)}\left(a+h_{l}+2 t+2 h_{c}\right)=u^{(5)}\left(a+h_{l}+2 t+2 h_{c}\right) \\
& \sigma_{r}^{(4)}\left(a+h_{l}+2 t+2 h_{c}\right)=\sigma_{r}^{(5)}\left(a+h_{l}+2 t+2 h_{c}\right) .
\end{aligned}
$$

The pipe is subjected to a combination of internal and external pressure (Fig. 1)

$$
\sigma_{r}^{(1)}(a)=-p_{i}, \quad \sigma_{r}^{(5)}(b)=-p_{o} .
$$

\subsection{Method of solution}

Due to the axial symmetry of the sandwich pipe and applied loading, we can consider elasticity problem as a plane strain one. In this case, the equilibrium equations, strain-displacement equations and stress-strain relations have the form

$$
\begin{gathered}
\frac{d}{d r} \sigma_{r}(r)+\frac{\sigma_{r}(r)-\sigma_{\theta}(r)}{r}=0, \\
\epsilon_{r}(r)=\frac{d}{d r} u(r), \quad \epsilon_{\theta}=\frac{u(r)}{r}, \\
\sigma_{r}(r)=\frac{E(r)}{(1+\nu)(1-2 \nu)}\left((1-\nu) \epsilon_{r}(r)+\nu \epsilon_{\theta}\right), \\
\sigma_{\theta}(r)=\frac{E(r)}{(1+\nu)(1-2 \nu)}\left((1-\nu) \epsilon_{\theta}(r)+\nu \epsilon_{r}\right) .
\end{gathered}
$$

By using the displacement formulation, the above equations can be reduced to Navier equation

$$
\frac{d^{2}}{d r^{2}} u(r)+\frac{1}{r} \frac{d}{d r} u(r)-\frac{u(r)}{r^{2}}+\frac{1}{E(r)} \frac{d}{d r} E(r)\left(\frac{d}{d r} u(r)+\frac{\nu}{1-\nu} \frac{u(r)}{r}\right)=0 .
$$

The specific form of Navier equation for layers 1 to 5 depends on the function that describes variation of Young's modulus with the radial co-ordinate within each layer. 


\section{$2.3 \quad$ Functionally graded materials (interlayers 2 and 4 )}

Substituting (2.1) that describes variation of Young's modulus with the radial co-ordinate within interlayer 2 into (2.9), we obtain

$$
\frac{d^{2}}{d r^{2}} u(r)+\frac{1}{r} \frac{d}{d r} u(r)-\frac{u(r)}{r^{2}}+\frac{n}{r}\left(\frac{d}{d r} u(r)+\frac{\nu}{1-\nu} \frac{u(r)}{r}\right)=0 .
$$

Solution of the above equation has the form

$$
u(r)=B_{1} r^{\alpha / 2-n / 2}+B_{2} r^{-\alpha / 2-n / 2},
$$

where

$$
\alpha=\frac{\sqrt{(\nu-1)\left((n+2)^{2} \nu-n^{2}-4\right)}}{\nu-1} .
$$

We remark that, as a consequence of Poissonś ratio constraint $0<\nu<1 / 2$, the sign of the term in the square root presents in the definition of (2.12) is always positive.

Substituting (2.11) into strain-displacement relations, (2.7), and then stress-strain relations, (2.8), the following expressions for radial and hoop stresses in the inner FGM interlayer are obtained

$$
\begin{aligned}
\sigma_{r}(r) & =\frac{r^{n / 2-1+\alpha / 2}((\nu-1) \alpha-(n+2) \nu+n) E_{l}}{2(1+\nu)(2 \nu-1)\left(a+h_{l}\right)^{n}} B_{1}+ \\
& -\frac{r^{n / 2-1-\alpha / 2}((\nu-1) \alpha+(n+2) \nu-n) E_{l}}{2(1+\nu)(2 \nu-1)\left(a+h_{l}\right)^{n}} B_{2}, \\
\sigma_{\theta}(r) & =-\frac{r^{n / 2-1+\alpha / 2}(\nu \alpha-(n+2) \nu+2) E_{l}}{2(1+\nu)(2 \nu-1)\left(a+h_{l}\right)^{n}} B_{1}+ \\
& +\frac{r^{n / 2-1-\alpha / 2}(\nu \alpha+(n+2) \nu-2) E_{l}}{2(1+\nu)(2 \nu-1)\left(a+h_{l}\right)^{n}} B_{2} .
\end{aligned}
$$

For the outer FGM interlayer, substituting equation (2.3) that describes variation of Young's modulus with the radial co-ordinate within layer 4 into Navier equation, (2.9), yields

$$
\frac{d^{2}}{d r^{2}} u(r)+\frac{1}{r} \frac{d}{d r} u(r)-\frac{u(r)}{r^{2}}-\frac{n}{2 a+H-r}\left(\frac{d}{d r} u(r)+\frac{\nu}{1-\nu} \frac{u(r)}{r}\right)=0 .
$$


This equation, after a rescaling and a translation, can be rewritten in the standard hypergeometric form. Its solutions can be found in (Abramowitz, M, Stegun, 1965). As a consequence of the physical meaning of the parameters involved in Eq.(2.14), we consider the following solutions:

$$
u(r)=D_{1} r \Theta_{1}(r)+D_{2}(2 a+H-r)^{1-n} r \Theta_{2}(r)
$$

where

$$
\begin{aligned}
& \Theta_{1}(r)={ }_{2} \mathrm{~F}_{1}\left(-\frac{\alpha}{2}+\frac{n}{2}+1, \frac{\alpha}{2}+\frac{n}{2}+1 ; n ; \frac{2 a+H-r}{2 a+H}\right) \\
& \Theta_{2}(r)={ }_{2} \mathrm{~F}_{1}\left(-\frac{\alpha}{2}-\frac{n}{2}+2, \frac{\alpha}{2}-\frac{n}{2}+2 ; 2-n ; \frac{2 a+H-r}{2 a+H}\right)
\end{aligned}
$$

are the hypergeometric functions.

We set also the quantities

$$
\begin{aligned}
& \Theta_{3}(r)={ }_{2} \mathrm{~F}_{1}\left(\frac{\alpha}{2}+\frac{n}{2}+2,-\frac{\alpha}{2}+\frac{n}{2}+2 ; n+1 ; \frac{2 a+H-r}{2 a+H}\right) \\
& \Theta_{4}(r)={ }_{2} \mathrm{~F}_{1}\left(\frac{\alpha}{2}-\frac{n}{2}+3,-\frac{\alpha}{2}-\frac{n}{2}+3 ; 3-n ; \frac{2 a+H-r}{2 a+H}\right)
\end{aligned}
$$

to write

$$
\begin{aligned}
& \frac{d}{d r} \Theta_{1}(r)=\frac{(\alpha / 2-n / 2-1)(\alpha / 2+n / 2+1) \Theta_{3}(r)}{n(2 a+H)} \\
& \frac{d}{d r} \Theta_{2}(r)=\frac{(\alpha / 2+n / 2-2)(\alpha / 2-n / 2+2) \Theta_{4}(r)}{(2-n)(2 a+H)}
\end{aligned}
$$

Substituting Eqs.(2.7) into and strain-displacement relations, Eq.(2.2b), and then stressstrain relations, Eqs. (2.2c), the following expressions for radial and hoop stress in the outer 
FGM interlayer (layer 4) are obtained as

$$
\begin{aligned}
& \sigma_{r}(r)=-\frac{(2 a+H-r)^{n} E_{l}}{(1+\nu)(2 \nu-1)\left(a+h_{l}\right)^{n}}\left(\frac{(2+n-\alpha)(2+n+\alpha)(\nu-1) r \Theta_{3}(r)}{4 n(2 a+H)}+\Theta_{1}(r)\right) D_{1}+ \\
& +\frac{E_{l}}{(1+\nu)(2 \nu-1)\left(a+h_{l}\right)^{n}}((n(\nu-1)-\nu+2) r-H-2 a) \Theta_{2}(r) D_{2}+ \\
& +\frac{E_{l}}{(1+\nu)(2 \nu-1)\left(a+h_{l}\right)^{n}} \frac{(2 a+H-r)(4-n+\alpha)(4-n-\alpha)(\nu-1) r \Theta_{4}(r)}{4(n-2)(2 a+H)} D_{2}, \\
& \sigma_{\theta}(r)=\frac{(2 a+H-r)^{n}}{(1+\nu)(2 \nu-1)\left(a+h_{l}\right)^{n}}\left(\frac{(2+n-\alpha)(2+n+\alpha) \nu r \Theta_{3}(r)}{4 n(2 a+H)}+\Theta_{1}(r)\right) D_{1}+ \\
& -\frac{E_{l}}{(1+\nu)(2 \nu-1)\left(a+h_{l}\right)^{n}}\left(((\nu(n-1)-1) r+2 a+H) \Theta_{2}(r)\right) D_{2}+ \\
& -\frac{E_{l}}{(1+\nu)(2 \nu-1)\left(a+h_{l}\right)^{n}} \frac{(2 a+H-r)(4-n+\alpha)(4-n-\alpha) \nu r \Theta_{4}(r)}{4(n-2)(2 a+H)} D_{2} .
\end{aligned}
$$

\subsection{Homogeneous material (layers 1, 3 and 5)}

For homogeneous layers, Young's modulus is constant through the layer thickness, and consequently Navier equation is reduced to

$$
\frac{d^{2}}{d r^{2}} u(r)+\frac{1}{r} \frac{d}{d r} u(r)-\frac{u(r)}{r^{2}}=0 .
$$

For the inner pipe (layer 1), the solution is written as:

$$
u(r)=A_{1} r+\frac{A_{2}}{r^{2}} .
$$

For the core layer (layer 3), the solutions is

$$
u(r)=C_{1} r+\frac{C_{2}}{r^{2}}
$$

For the outer pipe (layer 5), the solutions is

$$
u(r)=F_{1} r+\frac{F_{2}}{r^{2}}
$$

The arbitrary constants $A_{1}, A_{2}, B_{1}, B_{2}, C_{1}, C_{2}, D_{1}, D_{2}, F_{1}$ and $F_{2}$ are determined from the continuity conditions at the interfaces between the layers and the boundary conditions at the inner and outer surfaces of the pipe, (2.5). 
By setting

$$
\begin{aligned}
& T=a+h_{l}+t+2 h_{c}, \\
& Q=2\left(a+h_{l}\right)^{2} \nu-2 a\left(a+h_{l}\right)-h_{l}^{2}, \\
& M=\left(T-2 h_{c}\right)^{\alpha / 2}-\left(a+h_{l}\right)^{\alpha}\left(T-2 h_{c}\right)^{-\alpha / 2},
\end{aligned}
$$

we explicitly write some constants in the form

$$
\begin{aligned}
& A_{2}=-\frac{a^{2} A_{1}}{2 \nu-1}+\frac{(1+\nu) p_{i} a^{2}}{E_{l}}, \\
& B_{2}=\frac{Q A_{1}}{(2 \nu-1)\left(a+h_{l}\right)^{-\alpha / 2-n / 2+1}}-\left(a+h_{l}\right)^{\alpha} B_{1}+\frac{(\nu+1) p_{i} a^{2}}{E_{l}\left(a+h_{l}\right)^{-\alpha / 2-n / 2+1}}, \\
& C_{2}=\frac{Q\left(T-2 h_{c}\right)^{-\alpha / 2-n / 2+1} A_{1}}{(2 \nu-1)\left(a+h_{l}\right)^{-\alpha / 2-n / 2+1}}+M\left(T-2 h_{c}\right)^{-n / 2+1} B_{1}-\left(T-2 h_{c}\right)^{2} C_{1}+ \\
& +\frac{(\nu+1)\left(T-2 h_{c}\right)^{-\alpha / 2-n / 2+1} p_{i} a^{2}}{E_{l}\left(a+h_{l}\right)^{-\alpha / 2-n / 2+1}}, \\
& D_{2}=\frac{Q\left(T-2 h_{c}\right)^{-\alpha / 2+n / 2} A_{1}}{(2 \nu-1)\left(a+h_{l}\right)^{-\alpha / 2-n / 2+1} \Theta_{2}(T) T^{2}}+\frac{\left(T-2 h_{c}\right)^{n / 2} M B_{1}}{\Theta_{2}(T) T^{2}}+ \\
& +\frac{4 h_{c}\left(T-h_{c}\right) C_{1}}{\Theta_{2}(T)\left(T-2 h_{c}\right)^{-n+1} T^{2}}-\frac{\Theta_{1}(T) D_{1}}{\Theta_{2}(T)\left(T-2 h_{c}\right)^{-n+1}}+\frac{(\nu+1)\left(T-2 h_{c}\right)^{-\alpha / 2+n / 2} p_{i} a^{2}}{E_{l}\left(a+h_{l}\right)^{-\alpha / 2-n / 2+1} \Theta_{2}(T) T^{2}}, \\
& F_{2}=-\frac{(a+H)^{2} F_{1}}{2 \nu-1}+\frac{(\nu+1)(a+H)^{2} p_{o}}{E_{l}} .
\end{aligned}
$$

The remaining constants $A_{1}, B_{1}, C_{1}, D_{1}$ and $F_{1}$ are obtained by solving the following system

$$
\begin{aligned}
& c_{11} A_{1}+c_{12} B_{1}+c_{10}=0 \\
& c_{21} A_{1}+c_{22} B_{1}+c_{23} C_{1}+c_{20}=0 \\
& c_{31} A_{1}+c_{32} B_{1}+c_{33} C_{1}+c_{34} D_{1}+c_{30}=0 \\
& c_{41} A_{1}+c_{42} B_{1}+c_{43} C_{1}+c_{44} D_{1}+c_{40}=0 \\
& c_{51} A_{1}+c_{52} B_{1}+c_{53} C_{1}+c_{54} D_{1}+c_{55} F_{1}+c_{50}=0
\end{aligned}
$$

where the coefficients $c_{i j}$ are written in the Appendix. 


\subsection{Sandwich pipe without FGM interlayers}

As a reference case for comparison purposes, sandwich pipe without FGM interlayers will be considered. Solution for the reference case can be obtained from the solution presented above if both interlayers (layers 2 and 4 ) are assumed to be homogeneous isotropic materials with the same properties as the core (layer 3 ).

\section{NUMERICAL RESULTS AND DISCUSSION}

In this section, we consider a sandwich pipe, in which the inner and outer pipes are made of steel with Young's modulus of $200 \mathrm{GPa}$, while the core material is taken to be a polymer with Young's modulus of 1 GPa. Poisson's ratio is assumed to be equal to 0.3 for all layers. Geometrical parameters of the pipe are taken as follows: internal radius $a=0.1 \mathrm{~m}$, outer radius $b=0.15 \mathrm{~m}$, wall thickness of the inner and outer pipes $h_{l}=0.005 \mathrm{~m}$, interlayer thickness $t=0.005 \mathrm{~m}$, core thickness $0.03 \mathrm{~m}$. For this configuration, the inhomogeneity parameter in the power law for Young's modulus, Eq. (2.3) is equal to $n=-113.89$.

Figures 3, 4 and 5 show variation of respectively radial displacement, radial stress and hoop stress through the wall thickness of the sandwich pipe. The pipe is subjected to internal pressure of $10 \mathrm{MPa}$ or external pressure of $15 \mathrm{MPa}$. The results for the sandwich pipe with FGM interlayers are plotted with solid lines while results for the reference sandwich pipe without FGM interlayers are plotted with dashed lines.

In sandwich pipes with FGM interlayers, reduction of radial displacement compared to the reference pipe is observed both under internal and external pressure (Fig. 3). The biggest reduction occurs in the pipes to which the pressure is applied, i.e. in the inner pipe in the case of internal pressure and in the outer pipe in the case of external pressure. The reduction is constant through the core.

The magnitude of radial stress in the vicinity of core/inner pipe and core/outer pipe interfaces is slightly increased by absolute magnitude if FGM interlayers are present (Fig. 4). This effect is more pronounced at the interface which is closest to the surface of pressure 
application.

The most significant effect of FGM interlayers is observed in the variation of hoop stress (Fig. 5). In sandwich pipes with FGM interlayers, hoop stress discontinuity is eliminated compared to the reference sandwich pipe without FGM interlayers. Significant reduction of hoop stress is observed in the pipe to which pressure is applied (inner pipe in the case of internal pressure, outer pipe in the case of external pressure).

Variation of radial displacement, radial stress and hoop stress through the wall thickness of the sandwich pipe subjected to a combination of internal and external pressure is shown in Fig. 6, 7 and 8. The ratio of external-to-internal pressure is denoted as eta and taken as $0.5 ; 1 ; 2 ; 3\left(p_{i}=1 \mathrm{MPa}\right)$. Using formulae provided in Arjomandi K, Taheri F (2011), it was established that in this pressure range, the pipe wall remains in the linear elastic region and does not collapse.

It can be seen from Fig. 6 and 8 that the benefit of using FGM interlayers is increasing with the increasing external-to-internal pressure ratio $(\eta)$. The biggest reduction of radial displacement and hoop stress in the outer pipe is observed for $\eta=3$.

\section{CONCLUSION}

In this paper, sandwich pipes incorporating two FGM interlayers between the core layer and inner/outer pipes have been analysed in the framework of plane strain axisymmetric elasticity problem. Closed-form analytical solutions were derived for such pipes subjected to internal and/or external pressure. Comparative analysis of stress and displacement fields in sandwich pipes with and without FGM interlayers revealed beneficial effect of FGM interlayers on pipe response. It was established that under external pressure FGM interlayers contribute to significant reduction of hoop stress in the outer pipe. This effect becomes more pronounced as the external-to-internal pressure ratio increases. 


\section{ACKNOWLEDGEMENTS}

Financial support of this research by the Royal Society of Edinburgh and the Italian Academy of Sciences under International Exchanges Bilateral Programme grant is gratefully acknowledged.

\section{Appendix}

The coefficients of the system (2.24) are:

$$
\begin{aligned}
& c_{10}=\frac{(\nu-1)(\alpha+n-2) p_{i} a^{2}}{2\left(a+h_{l}\right)^{2}(2 \nu-1)}, \\
& c_{11}=-\frac{E_{l}(\nu-1)\left(-h_{l}\left(h_{l}+2 a\right)(2 \nu-1)-2 a^{2}(\nu-1)\right) \alpha}{2\left(a+h_{l}\right)^{2}(2 \nu-1)^{2}(\nu+1)}+ \\
& -\frac{E_{l}(\nu-1)\left(\left((1-2 \nu) h_{l}\left(h_{l}+2 a\right)-2 a^{2}(\nu-1)\right) n+2(1-2 \nu) h_{l}\left(h_{l}+2 a\right)-4 a^{2} \nu\right)}{2\left(a+h_{l}\right)^{2}(2 \nu-1)^{2}(\nu+1)}, \\
& c_{12}=-\frac{E_{l}\left(a+h_{l}\right)^{\alpha / 2-n / 2-1}(\nu-1) \alpha}{(1+\nu)(2 \nu-1)}, \\
& c_{20}=\frac{\left(\left(T-2 h_{c}\right)^{n} E_{l}((1-\nu) \alpha-(2+n) \nu+n)\left(a+h_{l}\right)^{-n}+2 E_{c}(2 \nu-1)\right) p_{i} a^{2}}{2 E_{l}(2 \nu-1)\left(T-2 h_{c}\right)^{\alpha / 2+n / 2+1}\left(a+h_{l}\right)^{-\alpha / 2-n / 2+1}}
\end{aligned}
$$




$$
\begin{aligned}
& c_{21}=-\frac{E_{l}(\nu-1)\left(a+h_{l}\right)^{\alpha / 2-n / 2-1} Q \alpha}{2(2 \nu-1)^{2}(\nu+1)\left(T-2 h_{c}\right)^{\alpha / 2-n / 2+1}}+ \\
& +\frac{\left(-\left(T-2 h_{c}\right)^{n} E_{l}((n+2) \nu-n)\left(a+h_{l}\right)^{-n}+2 E_{c}(2 \nu-1)\right) Q}{2(2 \nu-1)^{2}(\nu+1)\left(T-2 h_{c}\right)^{\alpha / 2+n / 2+1}\left(a+h_{l}\right)^{-\alpha / 2-n / 2+1}}, \\
& c_{22}=\frac{E_{l}\left(T-2 h_{c}\right)^{\alpha / 2+n / 2-1}((\nu-1) \alpha-(n+2) \nu+n)}{2\left(a+h_{l}\right)^{n}(\nu+1)(2 \nu-1)}-\frac{E_{c}\left(T-2 h_{c}\right)^{-n / 2-1} M}{(\nu+1)} \\
& +\frac{E_{l}\left(T-2 h_{c}\right)^{-\alpha / 2+n / 2-1}((\nu-1) \alpha+(n+2) \nu-n)}{2(\nu+1)(2 \nu-1)\left(a+h_{l}\right)^{-\alpha+n}}, \\
& c_{23}=\frac{2 E_{c}(1-\nu)}{(\nu+1)(2 \nu-1)}, \\
& c_{30}=-\frac{E_{c}\left(T-2 h_{c}\right)^{-\alpha / 2-n / 2+1} p_{i} a^{2}}{T^{2} E_{l}\left(a+h_{l}\right)^{-\alpha / 2-n / 2+1}}-\frac{\left(((\nu-1) n-\nu) T+2 h_{c}\right)\left(T-2 h_{c}\right)^{-\alpha / 2+n / 2} p_{i} a^{2}}{(2 \nu-1) T^{2}\left(a+h_{l}\right)^{-\alpha / 2+n / 2+1}}+ \\
& +\frac{(\nu-1)(-\alpha-4+n)(-\alpha+4-n) \Theta_{4}(T) a^{2}\left(T-2 h_{c}\right)^{-\alpha / 2+n / 2+1} p_{i}}{8(2 \nu-1) T(n-2)\left(T-h_{c}\right) \Theta_{2}(T)\left(a+h_{l}\right)^{-\alpha / 2+n / 2+1}}, \\
& c_{31}=-\frac{E_{c} Q\left(T-2 h_{c}\right)^{-\alpha / 2-n / 2+1}}{(2 \nu-1)(\nu+1) T^{2}\left(a+h_{l}\right)^{-\alpha / 2-n / 2+1}}+ \\
& -\frac{E_{l}\left((n-1) T \nu-T n+2 h_{c}\right) Q\left(T-2 h_{c}\right)^{-\alpha / 2+n / 2}}{(2 \nu-1)^{2} T^{2}(\nu+1)\left(a+h_{l}\right)^{-\alpha / 2+n / 2+1}}+ \\
& +\frac{E_{l}(-1+\nu)(-\alpha-4+n)(-\alpha+4-n) \Theta_{4}(T) Q}{8 T(n-2)\left(T-h_{c}\right)(2 \nu-1)^{2}(\nu+1) \Theta_{2}(T)\left(T-2 h_{c}\right)^{\alpha / 2-n / 2-1}\left(a+h_{l}\right)^{-\alpha / 2+n / 2+1}}, \\
& c_{32}=-\frac{E_{c} M\left(T-2 h_{c}\right)^{-n / 2+1}}{(\nu+1) T^{2}}-\frac{E_{l} M\left(((\nu-1) n-\nu) T+2 h_{c}\right)}{(2 \nu-1)(\nu+1) T^{2}\left(T-2 h_{c}\right)^{-n / 2}\left(a+h_{l}\right)^{n}}+ \\
& +\frac{E_{l}(\nu-1)(\alpha+4-n)(\alpha-4+n) \Theta_{4}(T) M}{8\left(a+h_{l}\right)^{n} T(n-2)\left(T-h_{c}\right)(\nu+1)(2 \nu-1) \Theta_{2}(T)\left(T-2 h_{c}\right)^{-1-n / 2}}, \\
& c_{33}=\frac{E_{c}\left(T-2 h_{c}\right)^{2}}{(\nu+1) T^{2}}-\frac{E_{c}}{(\nu+1)(2 \nu-1)}+ \\
& -\frac{4 E_{l}\left(((n-1) \nu-n) T+2 h_{c}\right) h_{c}\left(T-h_{c}\right)\left(a+h_{l}\right)^{-n}}{\left(T-2 h_{c}\right)^{-n+1} T^{2}(\nu+1)(2 \nu-1)}+ \\
& +\frac{E_{l}(\nu-1)(-\alpha-4+n)(-\alpha+4-n) \Theta_{4}(T) h_{c}\left(a+h_{l}\right)^{-n}}{2 T(n-2)\left(T-2 h_{c}\right)^{-n}(\nu+1)(2 \nu-1) \Theta_{2}(T)},
\end{aligned}
$$




$$
\begin{aligned}
& c_{34}=\frac{E_{l} T(\nu-1) \Theta_{1}(T)\left(n-1-\frac{\left(T-2 h_{c}\right)(-\alpha-4+n)(-\alpha+4-n) \Theta_{4}(T)}{8(n-2)\left(T-h_{c}\right) \Theta_{2}(T)}\right)}{(\nu+1)(2 \nu-1)\left(a+h_{l}\right)^{n}\left(T-2 h_{c}\right)^{-n+1}}+ \\
& -\frac{\left(T-2 h_{c}\right)^{n} E_{l}(-\alpha+2+n)(-\alpha-2-n) T(\nu-1) \Theta_{3}(T)}{8\left(T-h_{c}\right) n(\nu+1)(2 \nu-1)\left(a+h_{l}\right)^{n}}, \\
& c_{40}=\frac{(\nu+1) a^{2}\left(T-2 h_{c}\right)^{-\alpha / 2+n / 2} \Theta_{2}(T+t)(T+t) p_{i}}{E_{l} \Theta_{2}(T) T^{2}\left(a+h_{l}\right)^{n / 2-\alpha / 2}}-\frac{\left(T+t+h_{l}\right)^{2}(\nu+1) p_{o}}{(T+t) E_{l},} \\
& c_{41}=\frac{Q\left(T-2 h_{c}\right)^{-\alpha / 2+n / 2} \Theta_{2}(T+t)(T+t)}{(2 \nu-1) \Theta_{2}(T) T^{2}\left(a+h_{l}\right)^{n / 2-\alpha / 2}}, \quad c_{42}=\frac{(T+t) \Theta_{2}(T+t)\left(T-2 h_{c}\right)^{n / 2} M}{T^{2} \Theta_{2}(T)\left(a+h_{l}\right)^{n-1}}, \\
& c_{43}=\frac{4 h_{c}\left(T-h_{c}\right) \Theta_{2}(T+t)(T+t)}{\Theta_{2}(T)\left(T-2 h_{c}\right)^{-n+1} T^{2}\left(a+h_{l}\right)^{n-1}} \\
& c_{44}=-\frac{\Theta_{2}(T+t)(T+t) \Theta_{1}(T)}{\Theta_{2}(T)\left(T-2 h_{c}\right)^{-n+1}\left(a+h_{l}\right)^{n-1}}+(T+t) \Theta_{1}(T+t) \\
& c_{45}=-T-t+\frac{\left(T+t+h_{l}\right)^{2}}{(2 \nu-1)(T+t)}, \\
& c_{50}=\frac{\left(((\nu-1) n-\nu)\left(a+h_{l}\right)+2(\nu-1)(n-1)\left(h_{c}+t\right)\right) \Theta_{2}(T+t) p_{i} a^{2}}{(2 \nu-1) \Theta_{2}(T) T^{2}\left(T-2 h_{c}\right)^{\alpha / 2-n / 2}\left(a+h_{l}\right)^{n / 2+1-\alpha / 2}}+ \\
& +\frac{(T+t)(\nu-1)\left(a+h_{l}\right)(\alpha+4-n)(-\alpha+4-n) \Theta_{4}(T+t)\left(T-2 h_{c}\right)^{-\alpha / 2+n / 2} p_{i} a^{2}}{8(2 \nu-1)(n-2)\left(T-h_{c}\right) \Theta_{2}(T) T^{2}\left(a+h_{l}\right)^{-\alpha / 2+n / 2+1}}+ \\
& +\frac{\left(\left(T+t+h_{l}\right)\right)^{2} p_{o}}{(T+t)^{2}} \\
& c_{51}=\frac{E_{l}\left((T+t)((n-1) \nu-n)+2 t+2 h_{c}\right) Q \Theta_{2}(T+t)}{(2 \nu-1)^{2} \Theta_{2}(T) T^{2}(\nu+1)\left(T-2 h_{c}\right)^{\alpha / 2-n / 2}\left(a+h_{l}\right)^{-\alpha / 2+n / 2+1}}+ \\
& +\frac{E_{l}(T+t)(\nu-1)\left(a+h_{l}\right)(\alpha+4-n)(-\alpha+4-n) Q \Theta_{4}(T+t)}{8(n-2)\left(T-h_{c}\right)(2 \nu-1)^{2} \Theta_{2}(T) T^{2}(\nu+1)\left(T-2 h_{c}\right)^{\alpha / 2-n / 2}\left(a+h_{l}\right)^{-\alpha / 2+n / 2+1}}, \\
& c_{52}=\frac{E_{l}\left(((\nu-1) n-\nu) T+(\nu-1) n t+(2-\nu) t+2 h_{c}\right) M \Theta_{2}(T+t)}{\Theta_{2}(T) T^{2}(\nu+1)(2 \nu-1)\left(T-2 h_{c}\right)^{-n / 2}\left(a+h_{l}\right)^{n}}+ \\
& +\frac{E_{l}(T+t)(\nu-1)\left(a+h_{l}\right)(\alpha+4-n)(-\alpha+4-n) M \Theta_{4}(T+t)}{8(n-2)\left(T-h_{c}\right) \Theta_{2}(T) T^{2}(\nu+1)(2 \nu-1)\left(T-2 h_{c}\right)^{-n / 2}\left(a+h_{l}\right)^{n}},
\end{aligned}
$$




$$
\begin{aligned}
& c_{53}=-\frac{E_{l} P\left(T-2 h_{c}\right)^{n-1}\left(a+h_{l}\right)^{-n} \Theta_{2}(T+t)}{\left((T+t)(n(\nu-1)-\nu)+2\left(t+h_{c}\right)\right)^{-1} \Theta_{2}(T) T^{2}(\nu+1)(2 \nu-1)}+ \\
& +\frac{E_{l} P\left(\alpha^{2}-(4-n)^{2}\right)(T+t)(\nu-1)\left(T-2 h_{c}\right)^{n-1}\left(a+h_{l}\right)^{1-n} \Theta_{4}(T+t)}{8(n-2)\left(T-h_{c}\right) \Theta_{2}(T) T^{2}(\nu+1)(2 \nu-1)}, \\
& c_{54}=-\frac{E_{l}(n(\nu-1)-\nu)\left((T+t)+2\left(t+h_{c}\right)\right) \Theta_{1}(T) \Theta_{2}(T+t)}{(\nu+1)(2 \nu-1) \Theta_{2}(T)\left(a+h_{l}\right)^{n}}+ \\
& +\frac{E_{l}(T+t)(\nu-1)\left(a+h_{l}\right)\left(\alpha^{2}-(4-n)^{2}\right) \Theta_{4}(T+t) \Theta_{1}(T)}{8(n-2)\left(T-h_{c}\right)\left(T-2 h_{c}\right)^{-n+1}(\nu+1)(2 \nu-1) \Theta_{2}(T)\left(a+h_{l}\right)^{n}}+ \\
& -\frac{E_{l} \Theta_{1}(T+t)}{(\nu+1)(2 \nu-1)}+\frac{E_{l}\left(\alpha^{2}-(2+n)^{2}\right)(T+t)(\nu-1) \Theta_{3}(T+t)}{8\left(T-h_{c}\right) n(\nu+1)(2 \nu-1)}, \\
& c_{55}=-\frac{E_{l} h_{l}\left(2 T+2 t+h_{l}\right)}{(\nu+1)(T+t)^{2}(2 \nu-1)},
\end{aligned}
$$

where we have set

$$
P=\left(\left(T-2 h_{c}\right) T-a^{2}-2\left(t+3 h_{c}+h_{l}\right) a-4 h_{c}{ }^{2}-6\left(t+h_{l}\right) h_{c}-\left(t+h_{l}\right)^{2}\right) .
$$




\section{References}

Abramowitz, M, Stegun, I (1965) Handbook of Mathematical Functions. New York, Dover Publication.

An C, Duan M, Toledo Filho RD, Estefen SF (2014) Collapse of sandwich pipes with PVA fiber reinforced cementitious composites core under external pressure. Ocean Engineering 82, 1-13.

Arjomandi K, Taheri F (2010) Elastic buckling capacity of bonded and unbonded sandwich pipes under external hydrostatic pressure. Journal of Mechanics of Materials and Structures $\mathbf{5}(3), 391-408$.

Arjomandi K, Taheri F (2011a) Stability and post-buckling response of sandwich pipes under hydrostatic pressure. International Journal of Pressure Vessels and Piping 88, 138-148.

Arjomandi K, Taheri F (2011b) A new look at the external pressure capacity of sandwich pipes. Marine Structures 24, 23-42.

Arjomandi K, Taheri F (2011c) The influence of intra-layer adhesion configuration of the pressure capacity and optimized configuration of sandwich pipes. Ocean Engineering 38, 1869-1882.

Arjomandi K, Taheri F (2012) Bending capacity of sandwich pipes. Ocean Engineering 48, $17-31$.

Atashipour SA, Sburlati R, Atashipour SR (2014) Elastic analysis of thick-walled pressurized spherical vessels coated with functionally graded materials. Meccanica 49, 2965-2978

Bai Q, Bai Y (2014) Subsea Pipeline Design, Analysis, and Installation. Elsevier, Amsterdam.

Castello X, Estefen SF (2007) Limit strength and reeling effect of sandwich pipes with bonded layers. International Journal of Mechanical Sciences 49, 577-588.

Denniel S, Bonneau P, Savvy P-A (2011) Electrically heated pipe-in-pipe A qualified reelable technology to meet flow assurance challenges. Offshore 71(9). 
Denniel S (2015). Pipe-in-pipe technology adapts to changing needs in deep and shallow water. Offshore $\mathbf{7 5}(2)$.

Estefen SF, Netto TA, Pasqualino IP (2005) Strength analyses of sandwich pipes for ultra deepwaters. ASME Journal of Applied Mechanics 72, 599-608.

He T, Duan M, Wang J, Lv S, An C (2015) On the external pressure capacity of deep water sandwich pipes with inter-layer adhesion conditions. Applied Ocean Research 52, 115-124. Jha DK, Kant T, Singh RK (2013) A critical review of recent research on functionally graded plates. Composite Structures 96, 833-849.

Kashtalyan M, Menshykova M (2009) Effect of a functionally graded interlayer on threedimensional elastic deformation of coated plates subjected to transverse loading. Composite Structures 89(2), 167-176.

Sburlati R (2012) Analytical elastic solutions for pressurized hollow cylinders with internal functionally graded coatings. Composite Structures 94, 3592-3600.

Sburlati R. (2013) Stress concentration factor due to a functionally graded ring around a hole in an isotropic plate. International Journal of Solids and Structures 50, 3649-3658.

Sofiyev, A.H. (2014) The vibration and buckling of sandwich cylindrical shells covered by different coatings subjected to the hydrostatic pressure. Composite Structures 117, 124-134. Sofiyev, A.H. and Kuruoglu (2015a) Parametric instability of shear deformable sandwich cylindrical shells containing an FGM core under static and time dependent periodic axial loads. International Journal of Mechanical Sciences 101-102,114-123.

Sofiyev, A.H. and Kuruoglu (2015b) Dynamic instability of three-layered cylindrical shells containing an FGM interlayer. Thin Walled Structures 93, 10-21. 
Figure 1. Sketch of the mathematical problem studied

Figure 2. Young's modulus in the five layers of the pipe wall (three homogeneous layers and two FGM interlayers)

Figure 3. Radial displacement in the sandwich pipe wall with and without FGM interlayers

Figure 4. Radial stress in the sandwich pipe wall with and without FGM interlayers

Figure 5. Hoop stress in the sandwich pipe wall with and without FGM interlayers

Figure 6. Radial displacement for different ratio $\eta$

Figure 7. Radial stress for different ratio $\eta$

Figure 8. Hoop stress for different ratio $\eta$ 


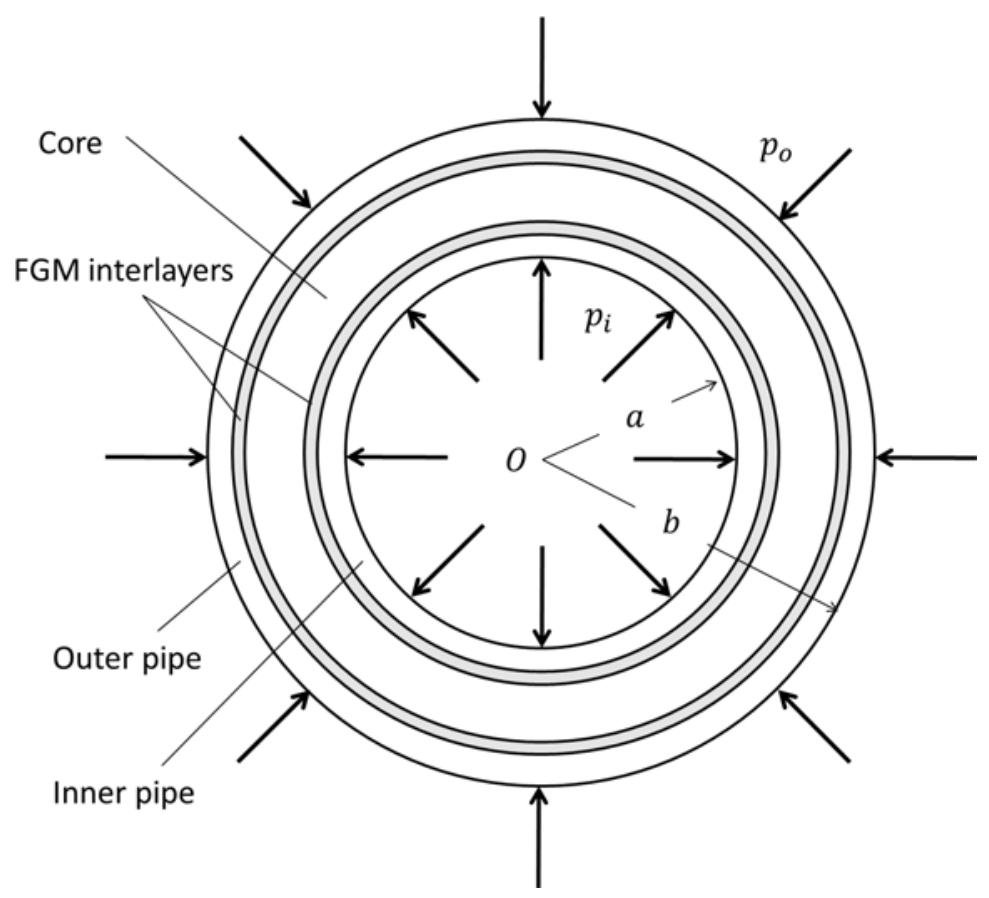

Figure 1: Sketch of the mathematical problem studied 


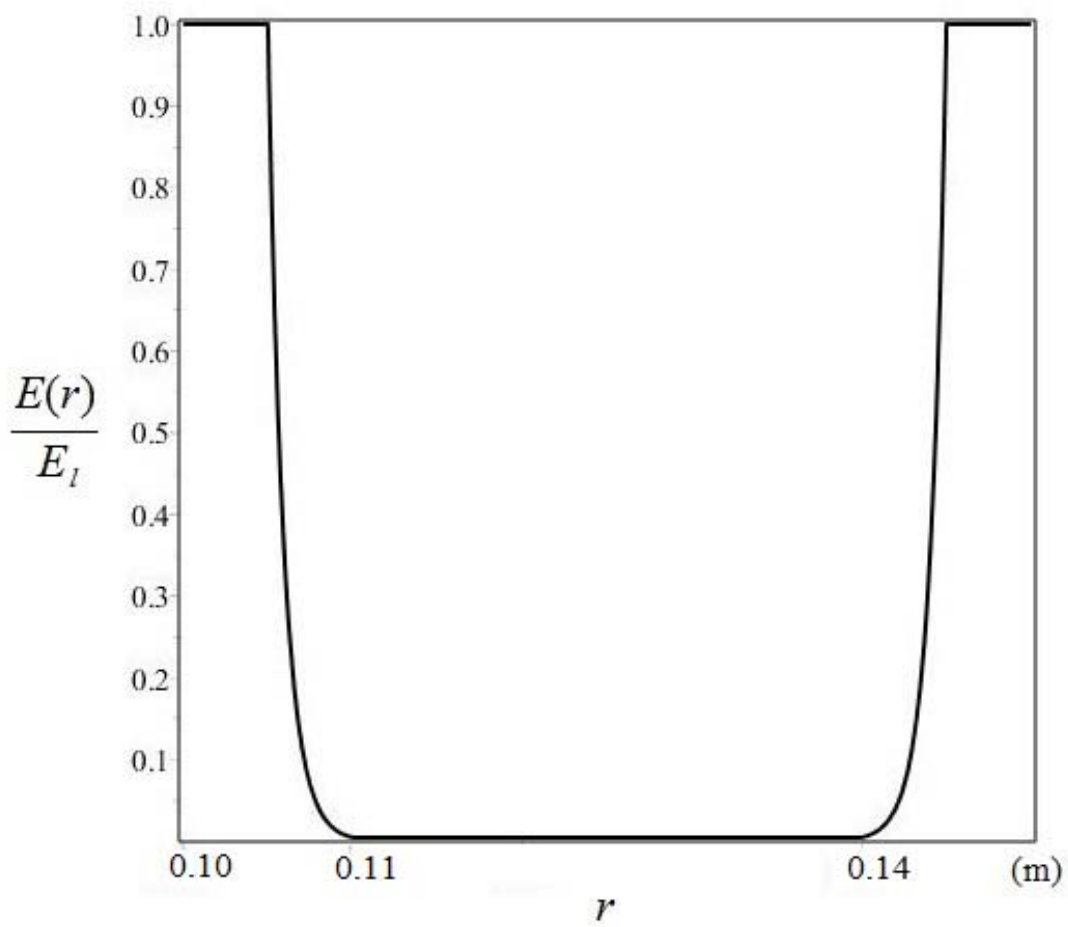

Figure 2: Young's modulus in the five layers of the pipe wall (three homogeneous layers and two FGM interlayers) 


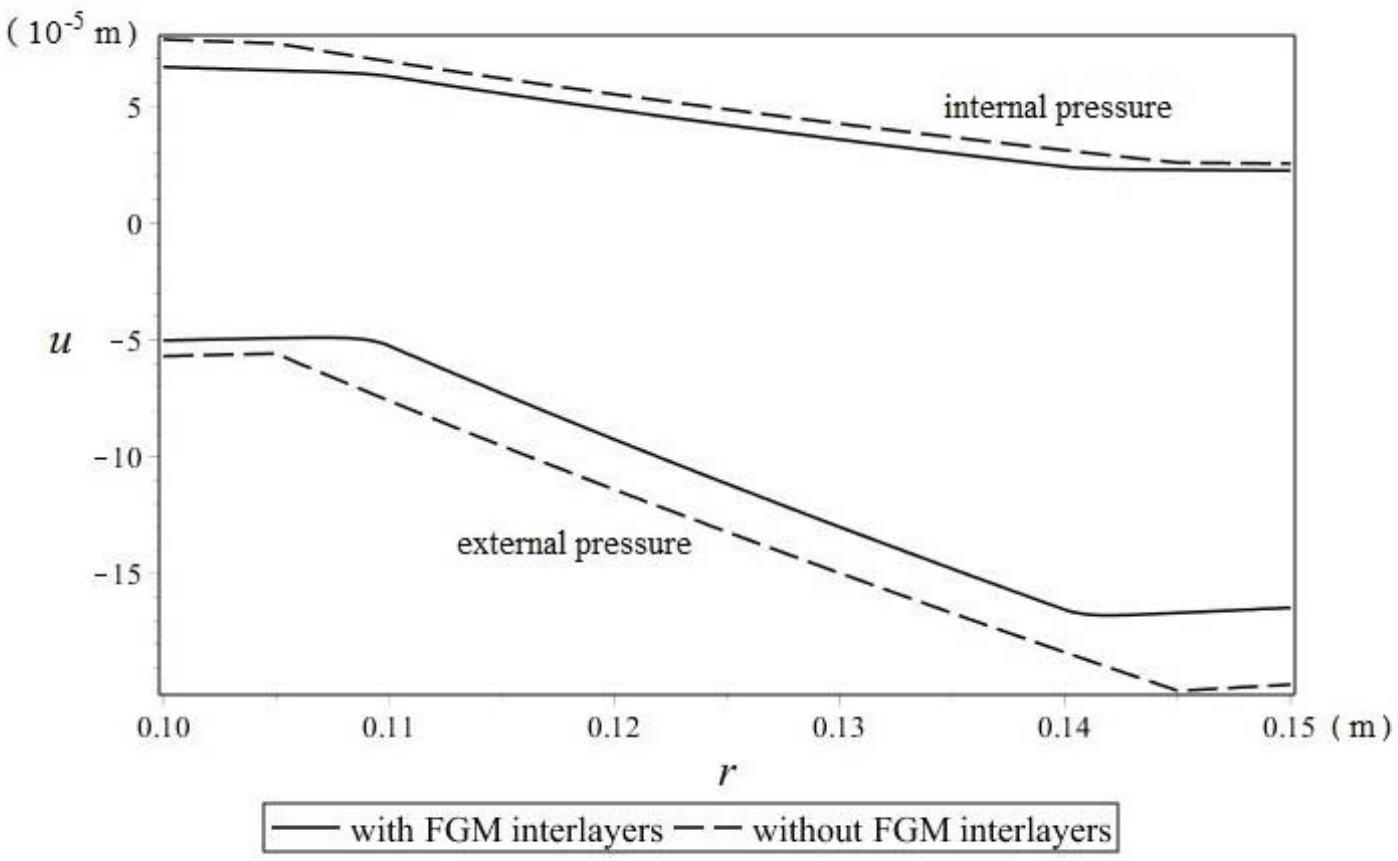

Figure 3: Radial displacement in the pipe with and without FGM interlayers 


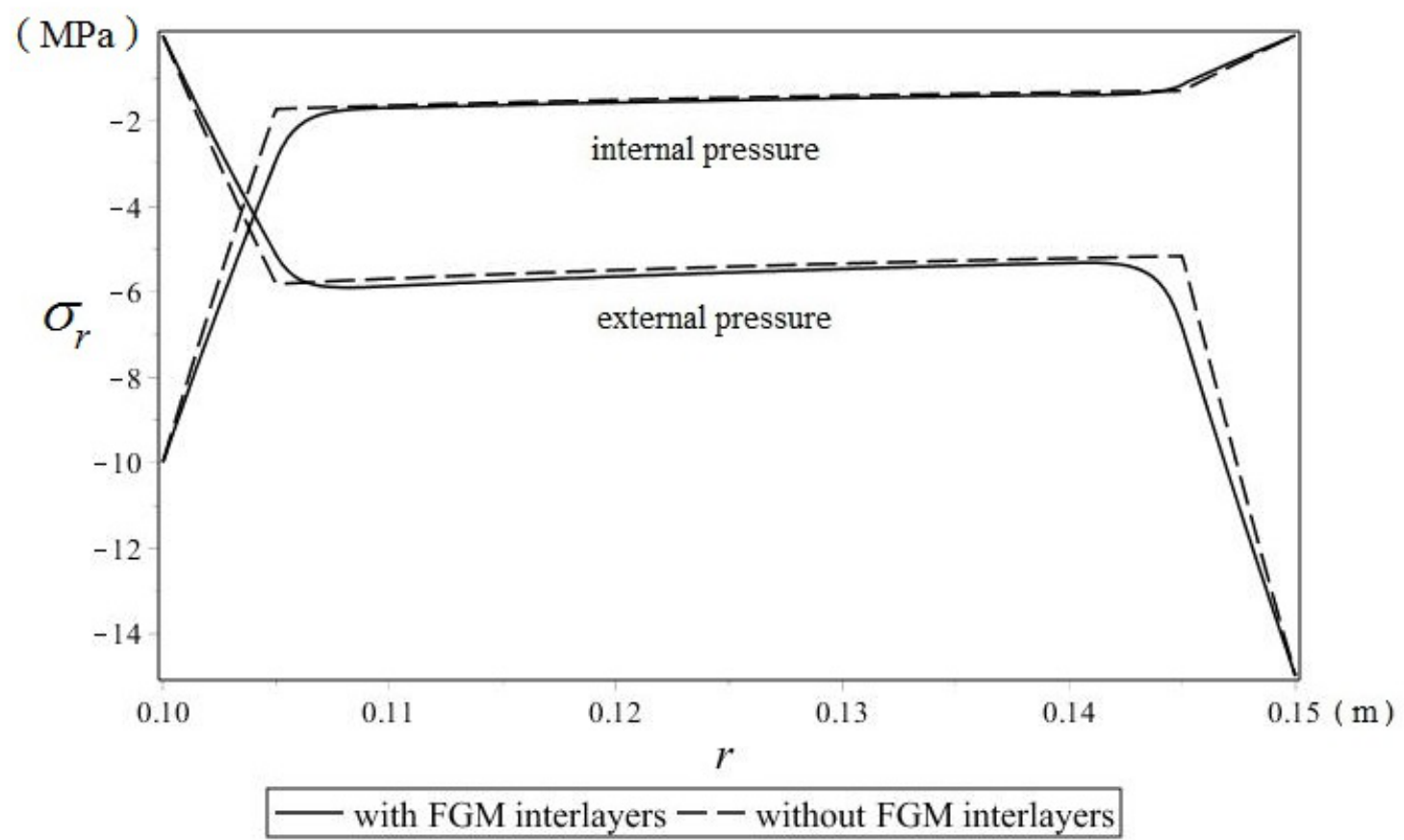

Figure 4: Radial stress in the pipe with and without FGM interlayers 


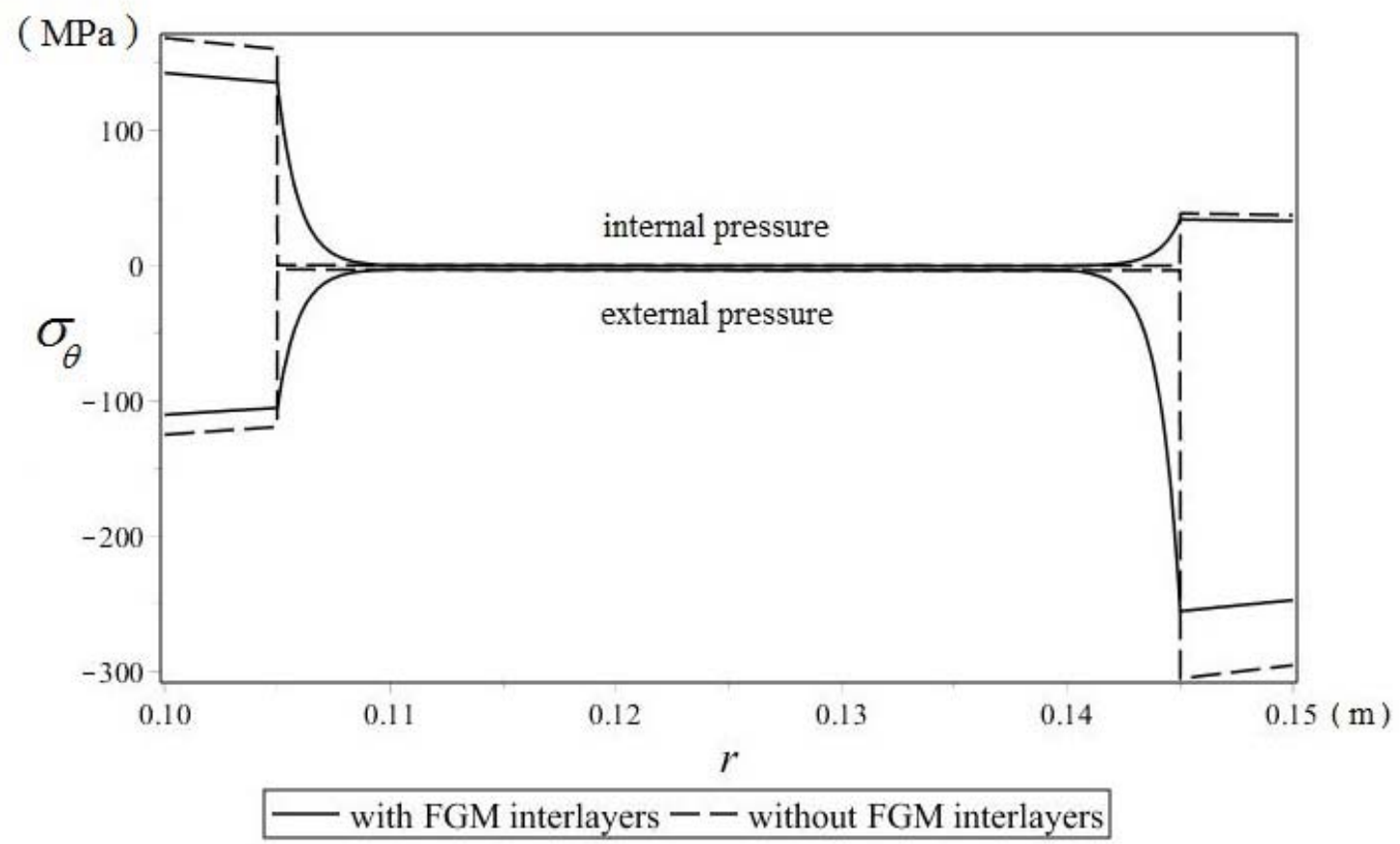

Figure 5: Hoop stress in the sandwich pipe wall with and without FGM interlayers 


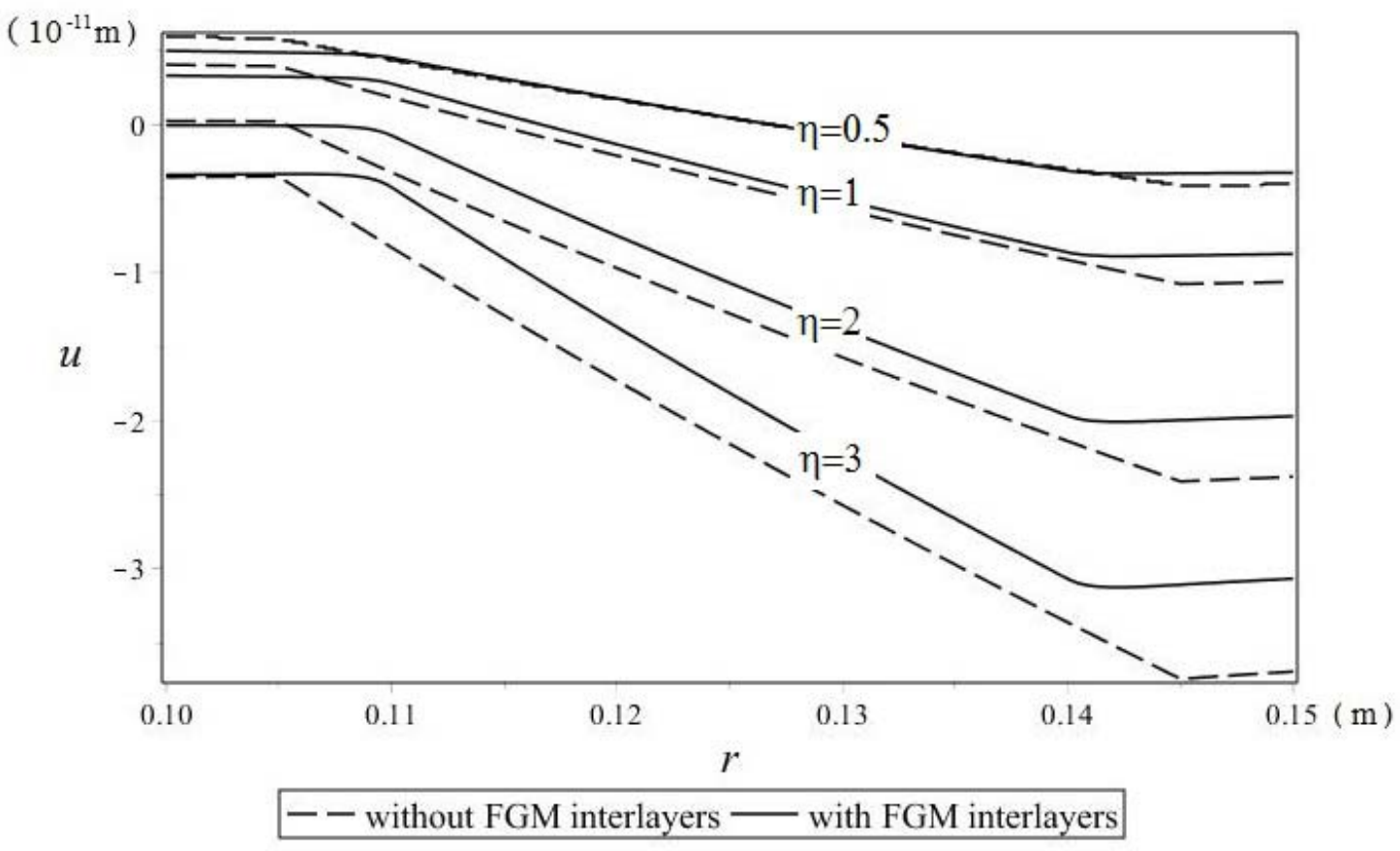

Figure 6: Radial displacement for different ratio $\eta$ 


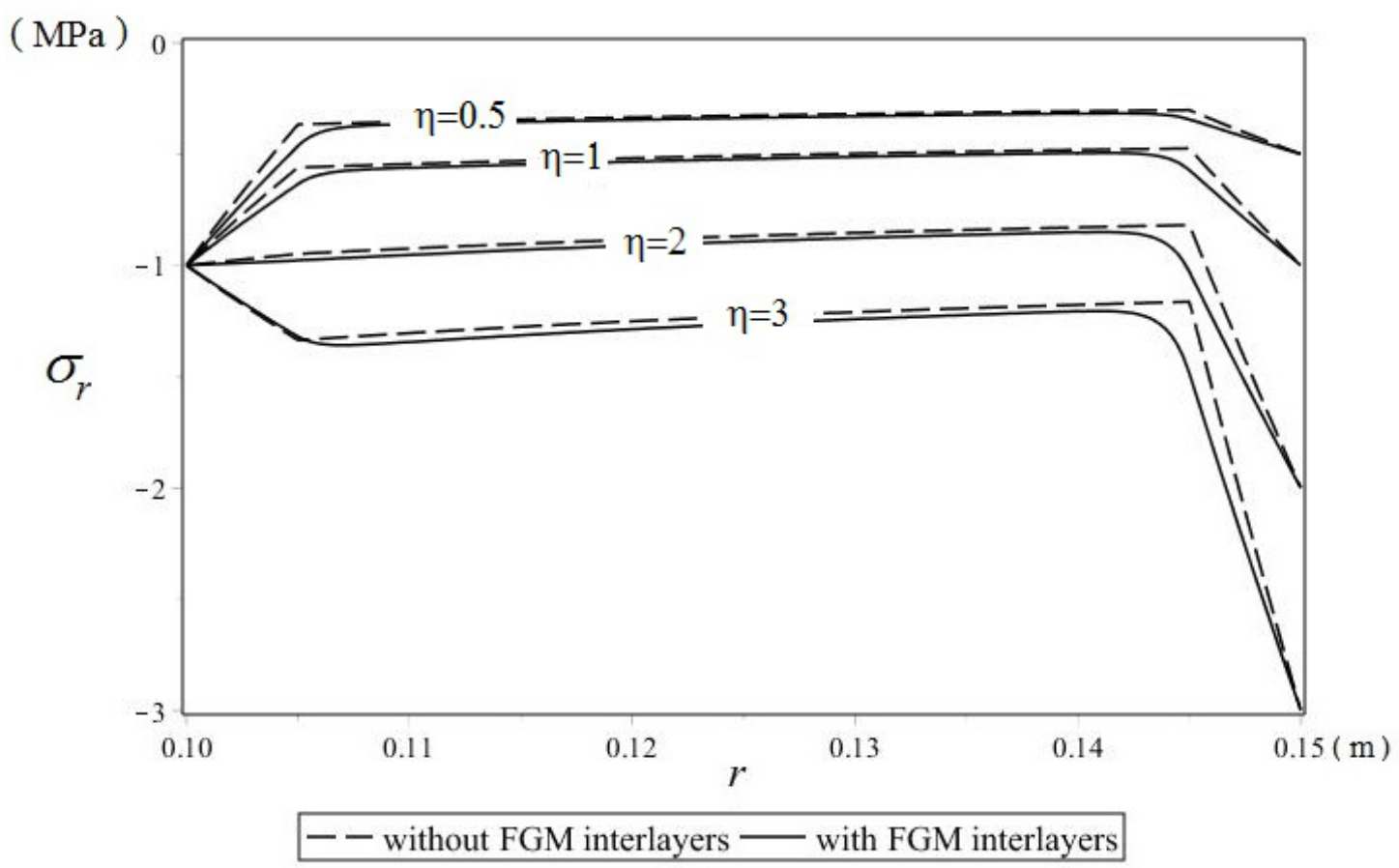

Figure 7: Radial stress for different ratio $\eta$ 


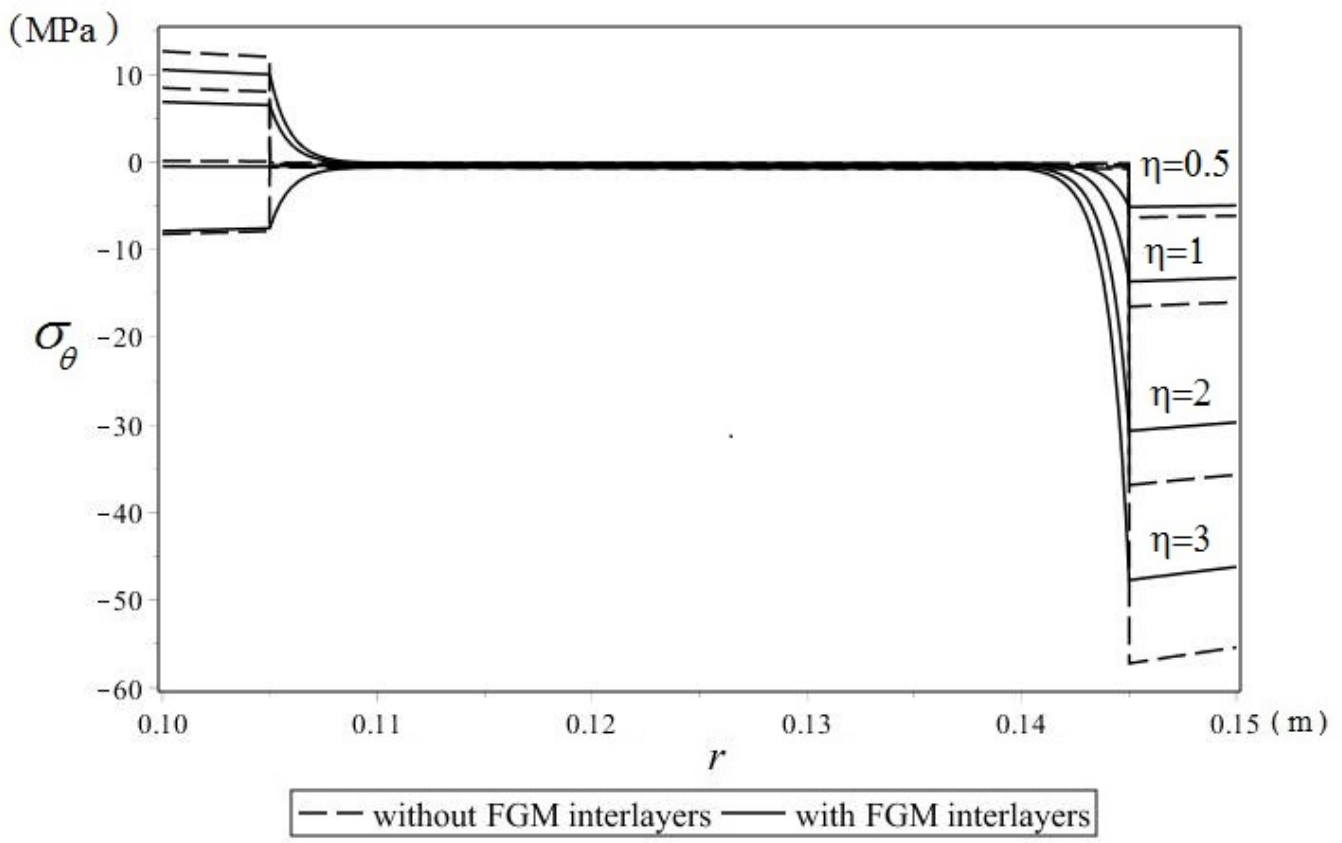

Figure 8: Hoop stress for different ratio $\eta$ 